\title{
Coherence and complementarity based on modified generalized skew information
}

\author{
Zhaoqi $\mathrm{Wu}^{1,4}$, Lin Zhang ${ }^{2,4 *}$, Shao-Ming $\mathrm{Fei}^{\dagger 3,4}$, Xianqing Li-Jost ${ }^{4}$ \\ 1. Department of Mathematics, Nanchang University, Nanchang 330031, P R China \\ 2. Institute of Mathematics, Hangzhou Dianzi University, Hangzhou 310018, P R China \\ 3. School of Mathematical Sciences, Capital Normal University, Beijing 100048, P R China \\ 4. Max-Planck-Institute for Mathematics in the Sciences, 04103 Leipzig, Germany
}

\begin{abstract}
We introduce modified generalized Wigner-Yanase-Dyson (MGWYD) skew information and modified weighted generalized Wigner-Yanase-Dyson (MWGWYD) skew information. By revisiting state-channel interaction based on MGWYD skew information, a family of coherence measures with respect to quantum channels is proposed. Furthermore, explicit analytical expressions of these coherence measures of qubit states are derived with respect to different quantum channels. Moreover, complementarity relations based on MGWYD skew information and MWGWYD skew information are also presented. Specifically, the conservation relations are investigated, while two interpretations of them including symmetry-asymmetry complementarity and wave-particle duality have been proposed.
\end{abstract}

PACS numbers: 03.65.Ud, 03.67.-a, 03.75.Gg

Key Words: Coherence; complementarity; modified generalized Wigner-Yanase-Dyson skew information; modified weighted generalized Wigner-Yanase-Dyson skew information

\section{Introduction}

Originating from the superposition principle, quantum coherence is a characteristic feature of quantum mechanics. Despite its wide applications in superconductivity, quantum thermodynamics and biological processes, the quantification of quantum coherence from a resource-theoretic perspective was initiated only recently [1]. Since then the study on quantum coherence has attracted much attention in recent years. Various kinds of coherence measures such as relative entropy of coherence, $l_{1}$ norm of coherence, intrinsic randomness of measurement, robustness of coherence, averaged coherence, max-relative entropy of coherence, modified trace distance, skew information, Hellinger distance, affin-

\footnotetext{
*Corresponding author. E-mail: godyalin@163.com;linzhang@mis.mpg.de

${ }^{\dagger}$ Corresponding author. E-mail: feishm@cnu.edu.cn
} 
ity distance and generalized $\alpha$-z-relative Rényi entropy have been proposed to quantify quantum coherence [2 15]. Coherence-generating power of quantum channels has also been investigated [16 18].

On the other hand, the relationships between quantum coherence and other quantum resources such as quantum entanglement, quantum discord and asymmetry [19 26] have been extensively studied. It has been shown that quantum coherence and asymmetry cannot be broadcasted [27, 28]. Quantum coherence is also tightly related to the optimization of quantum observables [29]. Recently, a regime of defining coherence measures by making use of POVMs has been put forward [30]. Utilizing the concept of resource destroying maps, the authors in 31] have established a framework of coherence theory on the level of quantum operations.

Complementarity is another important quantum feature which has been extensively studied since the advent of quantum mechanics. It is well known that the Bohr's complementarity principle plays an indispensable role in the basic theory of quantum mechanics in the early days [32]. This was manifested in wave-particle duality and uncertainty relations by many authors 33,42$]$.

Recently, by decomposing the state-channel interaction into a symmetric and an asymmetric part, the authors in [43] formalized a quantitative symmetry-asymmetry complementarity relation. The asymmetric part is given based on a modified version of Wigner-Yanase skew information, in which a Hermitian operator is replaced by a bounded linear operator (not-necessarily-Hermitian), which can be interpreted as a measure of coherence with respect to a quantum channel. However, as a desired property of a quantum coherence measure, the strong monotonicity of this quantity is not proved in [43]. In 44, Li provided an alternative proof of the monotonicity based on the modified skew information via operator algebra approach, and derived the strong monotonicity.

The generalized Wigner-Yanase-Dyson skew information with parameters $\alpha$ and $\beta$ has been introduced in [45]. It would be interesting if such generalized Wigner-YanaseDyson skew information could be utilized to characterize quantum coherence, general state-channel interactions and complementarity relations among the coherence measures. In this work, we define correspondingly the modified generalized Wigner-Yanase-Dyson (MGWYD) skew information and the modified weighted generalized Wigner-YanaseDyson (MWGWYD) skew information. Based on the MGWYD skew information and MWGWYD skew information, we study the state-channel interactions. It is shown that the asymmetric part of the generalized state-channel couplings can be regarded as a family of coherence of states with respect to a channel. Complementarity relations based on MGWYD skew information and MWGWYD skew information are also derived with physical interpretations. Some concluding remarks are given in Section 6. Our results shed new light on the study of coherence, and give rise to a basic framework for quantitatively addressing symmetry-asymmetry complementarity. 


\section{Preliminaries}

Let $\mathcal{H}$ be a $d$-dimensional Hilbert space, and $\mathcal{B}(\mathcal{H}), \mathcal{S}(\mathcal{H})$ and $\mathcal{D}(\mathcal{H})$ the set of all bounded linear operators, Hermitian operators and density operators on $\mathcal{H}$, respectively. Usually, a state and a channel are mathematically described by a density operator (positive operator of trace 1) and a completely positive trace preserving (CPTP) map, respectively [46]. Nevertheless, in this paper when we discuss state-channel interactions, a channel is assumed to be a completely positive trace nonincreasing map, while a quantum operation is assumed to be a completely positive trace preserving (CPTP) map, in accord with Ref. [43].

Fix an orthonormal basis $\{|i\rangle\}_{i=1}^{d}$ of a $d$-dimensional Hilbert space $\mathcal{H}$. The density operators which are diagonal in this basis are called incoherent states and the set of all incoherent states is denoted by $\mathcal{I}$, i.e.,

$$
\left.\mathcal{I}=\left\{\delta \in \mathcal{D}(\mathcal{H})\left|\delta=\sum_{i} p_{i}\right| i\right\rangle\langle i|, p_{i} \geq 0, \quad \sum_{i} p_{i}=1\right\}
$$

Let $\Lambda$ be a completely positive trace preserving (CPTP) map

$$
\Lambda(\rho)=\sum_{n} K_{n} \rho K_{n}^{\dagger}
$$

where $K_{n}$ are Kraus operators satisfying $\sum_{n} K_{n}^{\dagger} K_{n}=I_{d}$ with $I_{d}$ the identity operator. $K_{n}$ are called incoherent Kraus operators if $K_{n}^{\dagger} \mathcal{I} K_{n} \in \mathcal{I}$ for all $n$, and the corresponding $\Lambda$ is called an incoherent operation.

In [1], the authors proposed the conditions that a well-defined coherence measure $C$ should satisfy:

$\left(C_{1}\right)$ (Faithfulness) $C(\rho) \geq 0$ and $C(\rho)=0$ iff $\rho$ is incoherent.

$\left(C_{2}\right)$ (Monotonicity) $C(\Lambda(\rho)) \leq C(\rho)$ for any incoherent operation $\Lambda$.

$\left(C_{3}\right)$ (Convexity) $C(\cdot)$ is a convex function of $\rho$, i.e.,

$$
\sum_{n} p_{n} C\left(\rho_{n}\right) \geq C\left(\sum_{n} p_{n} \rho_{n}\right)
$$

where $p_{n} \geq 0, \quad \sum_{n} p_{n}=1$.

$\left(C_{4}\right)$ (Strong monotonicity) $C(\cdot)$ does not increase on average under selective incoherent operations, i.e.,

$$
C(\rho) \geq \sum_{n} p_{n} C\left(\varrho_{n}\right)
$$

where $p_{n}=\operatorname{Tr}\left(K_{n} \rho K_{n}^{\dagger}\right)$ are probabilities and $\varrho_{n}=\frac{K_{n} \rho K_{n}^{\dagger}}{p_{n}}$ are the post-measurement states, $K_{n}$ are incoherent Kraus operators.

Now, we recall the concepts of different kinds of skew information. For a density operator $\rho \in \mathcal{D}(\mathcal{H})$ and an observable $A \in \mathcal{S}(\mathcal{H})$, the Wigner-Yanase (WY) skew infor- 
mation [47] is defined by

$$
I_{\rho}(A)=-\frac{1}{2} \operatorname{Tr}\left(\left[\rho^{\frac{1}{2}}, A\right]^{2}\right),
$$

where $[X, Y]:=X Y-Y X$ is the commutator of $X$ and $Y$. A more general quantity was suggested by Dyson,

$$
I_{\rho}^{\alpha}(A)=-\frac{1}{2} \operatorname{Tr}\left(\left[\rho^{\alpha}, A\right]\left[\rho^{1-\alpha}, A\right]\right), \quad 0 \leq \alpha \leq 1,
$$

which is now called the Wigner-Yanase-Dyson (WYD) skew information. The quantity in Eq. (2) was further generalized to [45]

$$
I_{\rho}^{\alpha, \beta}(A)=-\frac{1}{2} \operatorname{Tr}\left(\left[\rho^{\alpha}, A\right]\left[\rho^{\beta}, A\right] \rho^{1-\alpha-\beta}\right), \quad \alpha, \beta \geq 0, \alpha+\beta \leq 1,
$$

which is termed as generalized Wigner-Yanase-Dyson (GWYD) skew information. It is easy to see that when $\alpha+\beta=1$, Eq. (3) reduces to Eq. (2), and Eq. (2) reduces to Eq. (11) when $\alpha=\frac{1}{2}$.

Another generalization of WYD skew information was given in [48]

$$
K_{\rho}^{\alpha}(A)=-\frac{1}{2} \operatorname{Tr}\left(\left[\frac{\rho^{\alpha}+\rho^{1-\alpha}}{2}, A_{0}\right]^{2}\right), 0 \leq \alpha \leq 1,
$$

where $A_{0}=A-\operatorname{Tr}(\rho A) I$. We call $K_{\rho}^{\alpha}(A)$ the weighted Wigner-Yanase-Dyson skew information in the following. Noting that $I_{\rho}(A)=I_{\rho}\left(A_{0}\right)$ when $\alpha=\frac{1}{2}$, one sees that Eq. (44) also reduces to Eq. (11) in this case.

Remarkable properties of these quantities and their applications in quantum information theory have been revealed and explored during the past few years [49 58. Nevertheless, quantum gates [46], generalized quantum gates [59], the Kraus operators of a quantum channel [46] and many other operators are not necessarily Hermitian. Therefore, it is natural to consider the corresponding definitions of the different types of the skew information mentioned above for pseudo-Hermitian and/or PT-symmetric quantum mechanics 60 65].

For a density operator $\rho \in \mathcal{D}(\mathcal{H})$ and an operator $A \in \mathcal{B}(\mathcal{H})$ (not necessarily Hermitian), a generalization of the quantity in Eq. (11) is defined by [66]

$$
\left|I_{\rho}\right|(A)=-\frac{1}{2} \operatorname{Tr}\left(\left[\rho^{\frac{1}{2}}, A^{\dagger}\right]\left[\rho^{\frac{1}{2}}, A\right]\right),
$$

which we refer to modified Wigner-Yanase (MWY) skew information. Similarly, a generalization of the quantity in Eq. (2) is defined by [67]

$$
\left|I_{\rho}^{\alpha}\right|(A)=-\frac{1}{2} \operatorname{Tr}\left(\left[\rho^{\alpha}, A^{\dagger}\right]\left[\rho^{1-\alpha}, A\right]\right), \quad 0 \leq \alpha \leq 1,
$$

for any $A \in \mathcal{B}(\mathcal{H})$ and $\rho \in \mathcal{D}(\mathcal{H})$, which we call modified Wigner-Yanase-Dyson (MWYD) skew information. A generalization of the quantity in Eq. (4) is given by [68]

$$
\left|K_{\rho}^{\alpha}\right|(A)=-\frac{1}{2} \operatorname{Tr}\left(\left[\frac{\rho^{\alpha}+\rho^{1-\alpha}}{2}, A_{0}^{\dagger}\right]\left[\frac{\rho^{\alpha}+\rho^{1-\alpha}}{2}, A_{0}\right]\right), \quad 0 \leq \alpha \leq 1,
$$


for any $A \in L^{2}(H)$ and $\rho \in D(H)$, which we call modified weighted Wigner-Yanase-Dyson (MWWYD) skew information. The related quantity $\left|L_{\rho}^{\alpha}\right|(A)$ is defined by replacing the commutators in Eq. (7) by anti-commutators.

In addition, a Schatten p-norm [69] is defined as

$$
\|A\|_{p}=\left[\sum_{j=1}^{n}\left(s_{j}(A)\right)^{p}\right]^{1 / p},
$$

where $s_{j}(A)$ denotes the singular value of $A$. When $p=2$, it is called a Hilbert-Schmidt norm [69]. Note that the class of Schatten $p$-norms is a special type of unitarily invariant norms [69] satisfying $\||U A V\|=\|\|A\||$ for all $A \in M(n)$ and $U, V \in U(n)$, where $M(n)$ denotes the set of $n \times n$ matrices and $U(n)$ the unitary group in $M(n)$.

Recently, Luo et al. defined the following quantity for any operator $K \in \mathcal{B}(\mathcal{H})$ and state $\rho \in \mathcal{D}(\mathcal{H})$ [3],

$$
I(\rho, K)=\operatorname{Tr}\left(\left[\rho^{\frac{1}{2}}, K\right]^{\dagger}\left[\rho^{\frac{1}{2}}, K\right]\right)=\left\|\left[\rho^{\frac{1}{2}}, K\right]\right\|_{2}^{2},
$$

where $\|X\|_{2}^{2}=\operatorname{Tr}\left(X^{\dagger} X\right)$ is the Hilbert-Schmidt norm, $[X, Y]=\frac{1}{2}(X Y-Y X)$ is the commutator. This quantity is in fact the one defined in [66] (up to a constant factor). For the sake of convenience, we call it modified Wigner-Yanase (MWY) skew information in the following. Similarly, the following quantity was defined as a measure to quantify the symmetry between $\rho$ and $K$,

$$
J(\rho, K)=\operatorname{Tr}\left(\left\{\rho^{\frac{1}{2}}, K\right\}\left\{\rho^{\frac{1}{2}}, K\right\}^{\dagger}\right)=\left\|\left\{\rho^{\frac{1}{2}}, K\right\}\right\|_{2}^{2},
$$

where $\{X, Y\}=\frac{1}{2}(X Y+Y X)$ is the anti-commutator.

Any quantum channel (completely positive trace nonincreasing map) has the following Kraus representation

$$
\Phi(\rho)=\sum_{i} K_{i} \rho K_{i}^{\dagger}
$$

while the dual channel can be written as

$$
\Phi^{\dagger}(X)=\sum_{i} K_{i}^{\dagger} X K_{i}
$$

where $X$ is any non-negative operator.

With respect to Eqs. (8) and (9), for a quantum channel in the form of Eq. (10), it is defined that

$$
I(\rho, \Phi)=\sum_{i} I\left(\rho, K_{i}\right)
$$

and

$$
J(\rho, \Phi)=\sum_{i} J\left(\rho, K_{i}\right) .
$$


For any operator $K \in \mathcal{B}(\mathcal{H})$ and state $\rho \in \mathcal{D}(\mathcal{H})$, the following quantity 44 has been derived from the unified entropy

$$
I^{\alpha}(\rho, K)=\operatorname{Tr}\left(\left[\rho^{\alpha}, K\right]^{\dagger}\left[\rho^{1-\alpha}, K\right]\right), \quad 0 \leq \alpha \leq 1
$$

Note that this quantity is in fact the one defined in [67] (up to a constant factor). We shall call it modified Wigner-Yanase-Dyson ( $M W Y D$ ) skew information in the following. $J^{\alpha}(\rho, K)$ was defined by using anti-commutator in Eq. (12). Also, $I^{\alpha}(\rho, \Phi)$ and $J^{\alpha}(\rho, \Phi)$ have been proposed in a similar manner with the Kraus representation of a quantum channel.

\section{State-channel interactions based on modified generalized skew information}

In this section, we will study state-channel interaction based on modified generalized skew information. We first define the modified generalized Wigner-Yanase-Dyson $(M G W Y D)$ skew information for any operator $K \in \mathcal{B}(\mathcal{H})$ and state $\rho \in \mathcal{D}(\mathcal{H})$,

$$
I^{\alpha, \beta}(\rho, K)=\operatorname{Tr}\left(\left[\rho^{\alpha}, K\right]^{\dagger}\left[\rho^{\beta}, K\right] \rho^{1-\alpha-\beta}\right), \quad \alpha, \beta \geq 0, \alpha+\beta \leq 1,
$$

and the related quantity $J^{\alpha, \beta}(\rho, K)$,

$$
J^{\alpha, \beta}(\rho, K)=\operatorname{Tr}\left(\left\{\rho^{\alpha}, K\right\}^{\dagger}\left\{\rho^{\beta}, K\right\} \rho^{1-\alpha-\beta}\right), \quad \alpha, \beta \geq 0, \alpha+\beta \leq 1 .
$$

Note that when $\alpha+\beta=1, I^{\alpha, \beta}(\rho, K)$ and $J^{\alpha, \beta}(\rho, K)$ reduce to $I^{\alpha}(\rho, K)$ and $J^{\alpha}(\rho, K)$, respectively. Furthermore, corresponding to the map $\Phi(\rho)=\sum_{i} K_{i} \rho K_{i}^{\dagger}$, we define

$$
I^{\alpha, \beta}(\rho, \Phi)=\sum_{i} I^{\alpha, \beta}\left(\rho, K_{i}\right), \quad \alpha, \beta \geq 0, \alpha+\beta \leq 1
$$

and

$$
J^{\alpha, \beta}(\rho, \Phi)=\sum_{i} J^{\alpha, \beta}\left(\rho, K_{i}\right), \quad \alpha, \beta \geq 0, \alpha+\beta \leq 1 .
$$

On the other hand, we define the modified weighted generalized Wigner-YanaseDyson (MWGWYD) skew information for any operator $K \in \mathcal{B}(\mathcal{H})$ and state $\rho \in \mathcal{D}(\mathcal{H})$,

$$
V^{\alpha, \beta}(\rho, K)=\operatorname{Tr}\left(\left[\frac{\rho^{\alpha}+\rho^{\beta}}{2}, K\right]^{\dagger}\left[\frac{\rho^{\alpha}+\rho^{\beta}}{2}, K\right] \rho^{1-\alpha-\beta}\right), \quad \alpha, \beta \geq 0, \alpha+\beta \leq 1,
$$

with the related quantity

$$
W^{\alpha, \beta}(\rho, K)=\operatorname{Tr}\left(\left\{\frac{\rho^{\alpha}+\rho^{\beta}}{2}, K\right\}^{\dagger}\left\{\frac{\rho^{\alpha}+\rho^{\beta}}{2}, K\right\} \rho^{1-\alpha-\beta}\right), \quad \alpha, \beta \geq 0, \alpha+\beta \leq 1 .
$$

Obviously, when $\alpha+\beta=1, V^{\alpha, \beta}(\rho, K)$ and $W^{\alpha, \beta}(\rho, K)$ reduce to $\left|K_{\rho}^{\alpha}\right|(A)$ and $\left|L_{\rho}^{\alpha}\right|(A)$ defined in [68] (up to a constant factor), respectively. With respect to the map $\Phi(\rho)=$ $\sum_{i} K_{i} \rho K_{i}^{\dagger}$, we define

$$
V^{\alpha, \beta}(\rho, \Phi)=\sum_{i} V^{\alpha, \beta}\left(\rho, K_{i}\right), \quad \alpha, \beta \geq 0, \alpha+\beta \leq 1
$$


and

$$
W^{\alpha, \beta}(\rho, \Phi)=\sum_{i} W^{\alpha, \beta}\left(\rho, K_{i}\right), \quad \alpha, \beta \geq 0, \alpha+\beta \leq 1 .
$$

Remark 1 As noted in [43] and [4], we can also prove that the quantities $I^{\alpha, \beta}(\rho, \Phi)$, $J^{\alpha, \beta}(\rho, \Phi), V^{\alpha, \beta}(\rho, \Phi)$ and $W^{\alpha, \beta}(\rho, \Phi)$ are independent of the choice of the Kraus operators of $\Phi$, which guarantees that the quantities given by Eqs. (17), (18), (21) and (22) are all well-defined.

From the above definitions, we have the following results of $I^{\alpha, \beta}(\rho, K)$ (the $W^{\alpha, \beta}(\rho, K)$ admits similar properties).

Proposition 1 For $\alpha, \beta \geq 0, \alpha+\beta \leq 1, K \in \mathcal{B}(\mathcal{H})$ and $\rho \in \mathcal{D}(\mathcal{H})$, it holds that

(i) $I^{\alpha, \beta}(\rho, K)=I^{\beta, \alpha}(\rho, K)$;

(ii) If $\alpha, \beta \in[0,1], \alpha+2 \beta \leq 1$ and $2 \alpha+\beta \leq 1$, then $I^{\alpha, \beta}(\rho, K)$ is convex in $\rho$. In particular, $I^{\alpha}(\rho, K)$ is convex in $\rho$ for $0 \leq \alpha \leq 1$.

Proof. (i) follows immediately from the definition. It follows from [11] that (ii) holds when $K \in \mathcal{S}(\mathcal{H})$. It can be seen that (ii) also holds for any $K \in \mathcal{B}(\mathcal{H})$ from the proof in [53].

\section{A family of coherence measures of a state with respect to a channel}

In this section, we demonstrate that $I^{\alpha, \beta}(\rho, \Phi)$ could be viewed as a bona fide family of coherence measures of $\rho$ with respect to a channel $\Phi$ under certain conditions. We first prove the following properties of $I^{\alpha, \beta}(\rho, \Phi)$.

Theorem 1 For channel $\Phi(\rho)=\sum_{j} K_{j} \rho K_{j}^{\dagger}$, the quantity $I^{\alpha, \beta}(\rho, \Phi)$ defined in Eq. (17) for $\alpha, \beta \geq 0$ with $\alpha+\beta \leq 1$ has the following properties:

(i) $I^{\alpha, \beta}(\rho, \Phi) \geq 0$, with the equality holding if and only if $\Phi^{\dagger}\left(\rho^{\alpha}\right)=\rho^{\alpha}, \Phi^{\dagger}\left(\rho^{\beta}\right)=\rho^{\beta}$ and $\Phi^{\dagger}\left(\rho^{\alpha+\beta}\right)=\rho^{\alpha+\beta}$.

(ii) If $\alpha, \beta \in[0,1], \alpha+2 \beta \leq 1$ and $2 \alpha+\beta \leq 1$, then $I^{\alpha, \beta}(\rho, \Phi)$ is convex in $\rho$.

(iii) (Ancillary independence) $I^{\alpha, \beta}\left(\rho^{a} \otimes \rho^{b}, \Phi^{a} \otimes \mathbf{1}^{b}\right)=I^{\alpha, \beta}\left(\rho^{a}, \Phi^{a}\right)$, where $\rho^{a}$ and $\rho^{b}$ are any states of systems $a$ and $b$, respectively, and $\mathbf{1}^{b}$ is the identity channel on system $b$.

(iv) (Monotonicity) If a channel $\mathcal{E}$ admits the representation $\mathcal{E}(\rho)=\sum_{i} E_{i} \rho E_{i}^{\dagger}$, then

$$
I^{\alpha, \beta}(\mathcal{E}(\rho), \Phi) \geq I^{\alpha, \beta}(\rho, \Phi)
$$

provided that one of the following two conditions is satisfied for all $i$ :

(1) $\mathcal{E}^{\dagger}\left(K_{i}\right)=K_{i}, \mathcal{E}^{\dagger}\left(K_{i}^{\dagger} K_{i}\right)=K_{i}^{\dagger} K_{i}, \mathcal{E}^{\dagger}\left(K_{i} K_{i}^{\dagger}\right)=K_{i} K_{i}^{\dagger}$ and $\left[\rho^{1-\alpha-\beta}, K_{i}\right]=0$;

(2) $\mathcal{E}^{\dagger}\left(K_{i}\right)=K_{i}, \mathcal{E}^{\dagger}\left(K_{i}^{\dagger} K_{i}\right)=K_{i}^{\dagger} K_{i}$ and $\left[\rho^{\alpha+\beta}, K_{i}\right]=0$.

(v) (Strong monotonicity) If a channel $\mathcal{E}$ admits the representation $\mathcal{E}(\rho)=\sum_{i} E_{i} \rho E_{i}^{\dagger}$, 
then

$$
\sum_{i} p_{i} I^{\alpha, \beta}\left(\rho_{i}, \Phi\right) \geq I^{\alpha, \beta}(\rho, \Phi)
$$

where $p_{i}=\operatorname{Tr} E_{i} \rho E_{i}^{\dagger}$ and $\rho_{i}=E_{i} \rho E_{i}^{\dagger} / p_{i}$, provided that condition (1) or (2) in item (iv) is satisfied for all $i$ and $\alpha, \beta>0$ with $\alpha+\beta \leq 1$.

Proof. (i) and (ii) can be verified easily from the definitions and the property (ii) in Proposition 1. Direct calculation shows that

$$
\begin{aligned}
& 4 I^{\alpha, \beta}\left(\rho^{a} \otimes \rho^{b}, \Phi^{a} \otimes \mathbf{1}^{b}\right) \\
= & 1-\operatorname{Tr}\left[\left(\rho^{a} \otimes \rho^{b}\right)^{1-\alpha}\left(\Phi^{a \dagger} \otimes \mathbf{1}^{b}\right)\left(\left(\rho^{a} \otimes \rho^{b}\right)^{\alpha}\right)\right]-\operatorname{Tr}\left[\left(\rho^{a} \otimes \rho^{b}\right)^{1-\beta}\left(\Phi^{a \dagger} \otimes \mathbf{1}^{b}\right)\left(\left(\rho^{a} \otimes \rho^{b}\right)^{\beta}\right)\right] \\
& -\operatorname{Tr}\left[\left(\rho^{a} \otimes \rho^{b}\right)^{1-\alpha-\beta}\left(\Phi^{a \dagger} \otimes \mathbf{1}^{b}\right)\left(\left(\rho^{a} \otimes \rho^{b}\right)^{\alpha+\beta}\right)\right] \\
= & 1-\operatorname{Tr}\left[\left(\rho^{a}\right)^{1-\alpha} \otimes\left(\rho^{b}\right)^{1-\alpha}\left(\Phi^{a \dagger}\left(\left(\rho^{a}\right)^{\alpha}\right) \otimes\left(\rho^{b}\right)^{\alpha}\right)\right]-\operatorname{Tr}\left[\left(\rho^{a}\right)^{1-\beta} \otimes\left(\rho^{b}\right)^{1-\beta}\left(\Phi^{a \dagger}\left(\left(\rho^{a}\right)^{\beta}\right) \otimes\left(\rho^{b}\right)^{\beta}\right)\right] \\
& +\operatorname{Tr}\left[\left(\rho^{a}\right)^{1-\alpha-\beta} \otimes\left(\rho^{b}\right)^{1-\alpha-\beta}\left(\Phi^{a \dagger}\left(\left(\rho^{a}\right)^{\alpha+\beta}\right) \otimes\left(\rho^{b}\right)^{\alpha+\beta}\right)\right] \\
= & 1-\operatorname{Tr}\left[\left(\rho^{a}\right)^{1-\alpha}\left(\Phi^{a \dagger}\left(\left(\rho^{a}\right)^{\alpha}\right)\right)\right]-\operatorname{Tr}\left[\left(\rho^{a}\right)^{1-\beta}\left(\Phi^{a \dagger}\left(\left(\rho^{a}\right)^{\beta}\right)\right)\right]+\operatorname{Tr}\left[\left(\rho^{a}\right)^{1-\alpha-\beta}\left(\Phi^{a \dagger}\left(\left(\rho^{a}\right)^{\alpha+\beta}\right)\right)\right] \\
= & 4 I^{\alpha, \beta}\left(\rho^{a}, \Phi^{a}\right) .
\end{aligned}
$$

Hence item (iii) holds.

It follows from Eq. (15) that

$$
I^{\alpha, \beta}\left(\rho, K_{i}\right)=\frac{1}{4}\left[\operatorname{Tr}\left(\rho^{1-\alpha-\beta} K_{i}^{\dagger} \rho^{\alpha+\beta} K_{i}\right)+\operatorname{Tr}\left(\rho K_{i}^{\dagger} K_{i}\right)-\operatorname{Tr}\left(\rho^{1-\alpha} K_{i}^{\dagger} \rho^{\alpha} K_{i}\right)-\operatorname{Tr}\left(\rho^{1-\beta} K_{i}^{\dagger} \rho^{\beta} K_{i}\right)\right] .
$$

If $\left[\rho^{1-\alpha-\beta}, K_{i}\right]=0$, the above equation becomes

$$
I^{\alpha, \beta}\left(\rho, K_{i}\right)=\frac{1}{4}\left[\operatorname{Tr}\left(\rho K_{i} K_{i}^{\dagger}\right)+\operatorname{Tr}\left(\rho K_{i}^{\dagger} K_{i}\right)-\operatorname{Tr}\left(\rho^{1-\alpha} K_{i}^{\dagger} \rho^{\alpha} K_{i}\right)-\operatorname{Tr}\left(\rho^{1-\beta} K_{i}^{\dagger} \rho^{\beta} K_{i}\right)\right] .
$$

If $\left[\rho^{\alpha+\beta}, K_{i}\right]=0$, the above equation becomes

$$
I^{\alpha, \beta}\left(\rho, K_{i}\right)=\frac{1}{4}\left[2 \operatorname{Tr}\left(\rho K_{i}^{\dagger} K_{i}\right)-\operatorname{Tr}\left(\rho^{1-\alpha} K_{i}^{\dagger} \rho^{\alpha} K_{i}\right)-\operatorname{Tr}\left(\rho^{1-\beta} K_{i}^{\dagger} \rho^{\beta} K_{i}\right)\right] .
$$

For $p \in(0,1), f(x)=x^{p}$ is operator monotone as well as operator concave [69]. So in either case, one can prove the conclusion (iv) by following the same line of the proof of Theorem 4 in [44] under the assumptions of item (iv).

At last, since (iii) holds, imitating the proof of Theorem 4 in [44], one proves that (v) is also true.

Remark 2 By Lemma 3 in [44], it is observed that $\mathcal{E}^{\dagger}\left(K_{i}\right)=K_{i}, \mathcal{E}^{\dagger}\left(K_{i}^{\dagger} K_{i}\right)=K_{i}^{\dagger} K_{i}$ and $\mathcal{E}^{\dagger}\left(K_{i} K_{i}^{\dagger}\right)=K_{i} K_{i}^{\dagger}$ if and only if $\left[E_{i}, K_{j}\right]=0$ and $\left[E_{i}, K_{j}^{\dagger}\right]=0$ for all $i$ and $j$. The later one could be seen as the condition that the channel $\mathcal{E}$ does not disturb channel $\Phi$, which was proposed as the assumption in proving the monotonicity of $I(\rho, \Phi)$ in [43]. Taking this fact into consideration, it follows from Theorem 1 that $I^{\alpha, \beta}(\rho, \Phi)(\alpha, \beta \geq 0, \alpha+\beta \leq 1)$ could be viewed as a family of coherence measures with respect to a channel $\Phi$ under the restrictive conditions. 
Remark 3 Direct computation shows that property (iv) (ancillary independence) in Theorem 1 does not hold for $V^{\alpha, \beta}(\rho, \Phi)(\alpha, \beta \geq 0, \alpha+\beta \leq 1)$ unless $\alpha=\beta$. But the proof of strong monotonicity in Theorem 1 relies heavily on the property of the ancillary independence. Hence the method used in [44] fails, and we do not know whether $V^{\alpha, \beta}(\rho, \Phi)$ can be regarded as a family of coherence measures with respect to a channel in general. Note that $V^{\alpha, \alpha}(\rho, \Phi)=I^{\alpha, \alpha}(\rho, \Phi) \cdot V^{\alpha, \alpha}(\rho, \Phi)(0 \leq \alpha \leq 1)$ is just a special class of $I^{\alpha, \beta}(\rho, \Phi)(\alpha, \beta \geq 0, \alpha+\beta \leq 1)$.

Now we calculate $I^{\alpha, \beta}(\rho, \Phi)$ and $V^{\alpha, \beta}(\rho, \Phi)$ for qubit states with respect to different kinds of quantum channels. Consider the Pauli channel defined by

$$
\Phi(\rho)=\sum_{j=0}^{3} p_{j} \sigma_{j} \rho \sigma_{j}, \quad p_{j} \geq 0, \quad \sum_{j=0}^{3} p_{j}=1,
$$

where $\sigma_{0}=I$, and $\sigma_{i}, i=1,2,3$, are Pauli matrices. For a qubit state $\rho=\frac{1}{2}(\mathbf{1}+\mathbf{r} \cdot \sigma)$, where $\mathbf{r}=\left\{r_{1}, r_{2}, r_{3}\right\}$ and $\sigma=\left\{\sigma_{1}, \sigma_{2}, \sigma_{3}\right\}$, its eigenvalues are $\lambda_{1,2}=(1 \mp|\mathbf{r}|) / 2$, and

$$
\rho^{\alpha}=\left(\begin{array}{cc}
\frac{\lambda_{1}^{\alpha}+\lambda_{2}^{\alpha}}{2}+\frac{r_{3}\left(\lambda_{2}^{\alpha}-\lambda_{1}^{\alpha}\right)}{2|\mathbf{r}|} & \frac{\left(-r_{1}+i r_{2}\right)\left(\lambda_{1}^{\alpha}-\lambda_{2}^{\alpha}\right)}{2|\mathbf{r}|} \\
\frac{\left(-r_{1}-i r_{2}\right)\left(\lambda_{1}^{\alpha}-\lambda_{2}^{\alpha}\right)}{2|\mathbf{r}|} & \frac{\lambda_{1}^{\alpha}+\lambda_{2}^{\alpha}}{2}-\frac{r_{3}\left(\lambda_{2}^{\alpha}-\lambda_{1}^{\alpha}\right)}{2|\mathbf{r}|}
\end{array}\right) .
$$

Then we have

$$
I^{\alpha, \beta}(\rho, \Phi)=\frac{1}{4} \sum_{j=1}^{3} p_{j} \frac{|\mathbf{r}|^{2}-r_{j}^{2}}{|\mathbf{r}|^{2}}\left(\lambda_{1}^{\alpha}-\lambda_{2}^{\alpha}\right)\left(\lambda_{1}^{\beta}-\lambda_{2}^{\beta}\right)\left(\lambda_{1}^{1-\alpha-\beta}+\lambda_{2}^{1-\alpha-\beta}\right) .
$$

In particular, when $p_{1}=p_{2}=p_{3}=p$, the Pauli channel defined in Eq. (23) becomes the depolarizing channel, and we have

$$
I^{\alpha, \beta}(\rho, \Phi)=\frac{1}{2} p\left(\lambda_{1}^{\alpha}-\lambda_{2}^{\alpha}\right)\left(\lambda_{1}^{\beta}-\lambda_{2}^{\beta}\right)\left(\lambda_{1}^{1-\alpha-\beta}+\lambda_{2}^{1-\alpha-\beta}\right),
$$

which is an increasing function of $p$. Taking $p_{1}=p$ and $p_{2}=p_{3}=0$ in Eq. (23), we get the bit-flipping channel, and have

$$
I^{\alpha, \beta}(\rho, \Phi)=\frac{1}{4} \cdot \frac{p\left(r_{2}^{2}+r_{3}^{2}\right)}{|\mathbf{r}|^{2}}\left(\lambda_{1}^{\alpha}-\lambda_{2}^{\alpha}\right)\left(\lambda_{1}^{\beta}-\lambda_{2}^{\beta}\right)\left(\lambda_{1}^{1-\alpha-\beta}+\lambda_{2}^{1-\alpha-\beta}\right) .
$$

Setting $p_{1}=p_{2}=0$ and $p_{3}=p$ in Eq. (23), we obtain the phase-flipping channel, and get

$$
I^{\alpha, \beta}(\rho, \Phi)=\frac{1}{4} \cdot \frac{p\left(r_{1}^{2}+r_{2}^{2}\right)}{|\mathbf{r}|^{2}}\left(\lambda_{1}^{\alpha}-\lambda_{2}^{\alpha}\right)\left(\lambda_{1}^{\beta}-\lambda_{2}^{\beta}\right)\left(\lambda_{1}^{1-\alpha-\beta}+\lambda_{2}^{1-\alpha-\beta}\right) .
$$

For the (unital) amplitude damping channel $\Phi(\rho)=\sum_{j=1}^{2} K_{j} \rho K_{j}^{\dagger}$ with

$$
K_{1}=|0\rangle\langle 0|+\sqrt{1-q}| 1\rangle\left\langle 1\left|, \quad K_{2}=\sqrt{q}\right| 1\right\rangle\langle 1|, \quad 0 \leq q \leq 1,
$$

we have

$$
I^{\alpha, \beta}(\rho, \Phi)=\frac{1}{4} \cdot \frac{(1-\sqrt{1-q})\left(r_{1}^{2}+r_{2}^{2}\right)}{2|\mathbf{r}|^{2}}\left(\lambda_{1}^{\alpha}-\lambda_{2}^{\alpha}\right)\left(\lambda_{1}^{\beta}-\lambda_{2}^{\beta}\right)\left(\lambda_{1}^{1-\alpha-\beta}+\lambda_{2}^{1-\alpha-\beta}\right) .
$$


And for the (nonunital) amplitude damping channel $\Phi(\rho)=\sum_{j=1}^{2} K_{j} \rho K_{j}^{\dagger}$ with

$$
K_{1}=|0\rangle\langle 0|+\sqrt{1-q}| 1\rangle\left\langle 1\left|, \quad K_{2}=\sqrt{q}\right| 0\right\rangle\langle 1|, \quad 0 \leq q \leq 1,
$$

we have

$$
\begin{aligned}
I^{\alpha, \beta}(\rho, \Phi)= & \frac{1}{4}\left[\frac{(1-\sqrt{1-q})\left(r_{1}^{2}+r_{2}^{2}\right)+q r_{3}^{2}}{2|\mathbf{r}|^{2}}\left(\lambda_{1}^{1-\alpha-\beta}+\lambda_{2}^{1-\alpha-\beta}\right)\right. \\
& \left.+\frac{q r_{3}}{2|\mathbf{r}|}\left(\lambda_{1}^{1-\alpha-\beta}-\lambda_{2}^{1-\alpha-\beta}\right)\right]\left(\lambda_{1}^{\alpha}-\lambda_{2}^{\alpha}\right)\left(\lambda_{1}^{\beta}-\lambda_{2}^{\beta}\right) .
\end{aligned}
$$

In a similar way, we can also compute $V^{\alpha, \beta}(\rho, \Phi)$ for a qubit state $\rho=\frac{1}{2}(I+\mathbf{r} \cdot \sigma)$ with respect to different channels. For the Pauli channel $\Phi$ defined in Eq. (23), we have

$$
V^{\alpha, \beta}(\rho, \Phi)=\frac{1}{4} \sum_{j=1}^{3} p_{j} \frac{|\mathbf{r}|^{2}-r_{j}^{2}}{4|\mathbf{r}|^{2}}\left(\lambda_{1}^{\alpha}-\lambda_{2}^{\alpha}+\lambda_{1}^{\beta}-\lambda_{2}^{\beta}\right)^{2}\left(\lambda_{1}^{1-\alpha-\beta}+\lambda_{2}^{1-\alpha-\beta}\right) .
$$

For the depolarizing channel $\Phi$ (a special case when $p_{1}=p_{2}=p_{3}=p$ ), we have

$$
V^{\alpha, \beta}(\rho, \Phi)=\frac{1}{8} p\left(\lambda_{1}^{\alpha}-\lambda_{2}^{\alpha}+\lambda_{1}^{\beta}-\lambda_{2}^{\beta}\right)^{2}\left(\lambda_{1}^{1-\alpha-\beta}+\lambda_{2}^{1-\alpha-\beta}\right),
$$

which is also an increasing function of $p$. For the bit-flipping channel $\Phi$ (a special case when $p_{1}=p$ and $p_{2}=p_{3}=0$ ), we have

$$
V^{\alpha, \beta}(\rho, \Phi)=\frac{1}{4} \cdot \frac{p\left(r_{2}^{2}+r_{3}^{2}\right)}{4|\mathbf{r}|^{2}}\left(\lambda_{1}^{\alpha}-\lambda_{2}^{\alpha}+\lambda_{1}^{\beta}-\lambda_{2}^{\beta}\right)^{2}\left(\lambda_{1}^{1-\alpha-\beta}+\lambda_{2}^{1-\alpha-\beta}\right) .
$$

For the phase-flipping channel $\Phi$ (a special case when $p_{1}=p_{2}=0$ and $p_{3}=p$ ), we have

$$
V^{\alpha, \beta}(\rho, \Phi)=\frac{1}{4} \cdot \frac{p\left(r_{1}^{2}+r_{2}^{2}\right)}{4|\mathbf{r}|^{2}}\left(\lambda_{1}^{\alpha}-\lambda_{2}^{\alpha}+\lambda_{1}^{\beta}-\lambda_{2}^{\beta}\right)^{2}\left(\lambda_{1}^{1-\alpha-\beta}+\lambda_{2}^{1-\alpha-\beta}\right) .
$$

For the (unital) amplitude damping channel (28), we have

$$
V^{\alpha, \beta}(\rho, \Phi)=\frac{1}{4} \cdot \frac{(1-\sqrt{1-q})\left(r_{1}^{2}+r_{2}^{2}\right)}{8|\mathbf{r}|^{2}}\left(\lambda_{1}^{\alpha}-\lambda_{2}^{\alpha}+\lambda_{1}^{\beta}-\lambda_{2}^{\beta}\right)^{2}\left(\lambda_{1}^{1-\alpha-\beta}+\lambda_{2}^{1-\alpha-\beta}\right) .
$$

And for the (nonunital) amplitude damping channel (30), we have

$$
\begin{aligned}
V^{\alpha, \beta}(\rho, \Phi)= & \frac{1}{4}\left[\frac{(1-\sqrt{1-q})\left(r_{1}^{2}+r_{2}^{2}\right)+q r_{3}^{2}}{8|\mathbf{r}|^{2}}\left(\lambda_{1}^{1-\alpha-\beta}+\lambda_{2}^{1-\alpha-\beta}\right)\right. \\
& \left.+\frac{q r_{3}}{8|\mathbf{r}|}\left(\lambda_{1}^{1-\alpha-\beta}-\lambda_{2}^{1-\alpha-\beta}\right)\right]\left(\lambda_{1}^{\alpha}-\lambda_{2}^{\alpha}+\lambda_{1}^{\beta}-\lambda_{2}^{\beta}\right)^{2} .
\end{aligned}
$$

We can see that when $\alpha=\beta=\frac{1}{2}$, the above analytical expressions for a qubit state with respect to certain channels reduce to the corresponding ones in Ref. [43].

\section{Complementarity relations and information conservation}


In Section 3, we have defined $I^{\alpha, \beta}(\rho, \Phi)$ and $J^{\alpha, \beta}(\rho, \Phi)$ using commutator and anticommutator, which is in a dual fashion in some sense. Another kind of generalization of the quantities $V^{\alpha, \beta}(\rho, \Phi)$ and $W^{\alpha, \beta}(\rho, \Phi)$ have also been introduced. In this section, we discuss the relationships between these two sets of quantities.

Set

$$
C^{\alpha, \beta}(\rho, K)=\frac{1}{2}\left[\operatorname{Tr}\left(\rho^{1-\alpha} K^{\dagger} \rho^{\alpha} K\right)+\operatorname{Tr}\left(\rho^{1-\beta} K^{\dagger} \rho^{\beta} K\right)\right], \quad \alpha, \beta \geq 0, \alpha+\beta \leq 1 .
$$

It follows from Eqs. (15) and (16) that

$$
I^{\alpha, \beta}(\rho, K)=\frac{1}{4}\left[\operatorname{Tr}\left(\rho^{1-\alpha-\beta} K^{\dagger} \rho^{\alpha+\beta} K+K \rho K^{\dagger}\right)-2 C^{\alpha, \beta}(\rho, K)\right]
$$

and

$$
J^{\alpha, \beta}(\rho, K)=\frac{1}{4}\left[\operatorname{Tr}\left(\rho^{1-\alpha-\beta} K^{\dagger} \rho^{\alpha+\beta} K+K \rho K^{\dagger}\right)+2 C^{\alpha, \beta}(\rho, K)\right] .
$$

Noting that

$$
C^{\alpha, \beta}(\rho, K)=\frac{1}{2}\left(\left\|\rho^{\frac{\alpha}{2}} K \rho^{\frac{1-\alpha}{2}}\right\|_{2}^{2}+\left\|\rho^{\frac{\beta}{2}} K \rho^{\frac{1-\beta}{2}}\right\|_{2}^{2}\right) \geq 0,
$$

we have

$$
I^{\alpha, \beta}(\rho, K) \leq J^{\alpha, \beta}(\rho, K),
$$

and thus

$$
I^{\alpha, \beta}(\rho, \Phi) \leq J^{\alpha, \beta}(\rho, \Phi)
$$

For any channel $\Phi$ with Kraus representation in the form of Eq. (10), the quantities $I^{\alpha, \beta}(\rho, \Phi)$ and $J^{\alpha, \beta}(\rho, \Phi)$ can be rewritten as

$$
I^{\alpha, \beta}(\rho, \Phi)=\frac{1}{4} \operatorname{Tr}\left[\rho^{1-\alpha-\beta} \Phi^{\dagger}\left(\rho^{\alpha+\beta}\right)+\Phi(\rho)-\rho^{1-\alpha} \Phi^{\dagger}\left(\rho^{\alpha}\right)-\rho^{1-\beta} \Phi^{\dagger}\left(\rho^{\beta}\right)\right]
$$

and

$$
J^{\alpha, \beta}(\rho, \Phi)=\frac{1}{4} \operatorname{Tr}\left[\rho^{1-\alpha-\beta} \Phi^{\dagger}\left(\rho^{\alpha+\beta}\right)+\Phi(\rho)+\rho^{1-\alpha} \Phi^{\dagger}\left(\rho^{\alpha}\right)+\rho^{1-\beta} \Phi^{\dagger}\left(\rho^{\beta}\right)\right],
$$

which implies that for $\alpha, \beta \geq 0, \alpha+\beta \leq 1$,

$$
I^{\alpha, \beta}(\rho, \Phi)+J^{\alpha, \beta}(\rho, \Phi)=\frac{1}{2} \operatorname{Tr}\left(\rho^{1-\alpha-\beta} \Phi^{\dagger}\left(\rho^{\alpha+\beta}\right)+\Phi(\rho)\right) .
$$

When $\alpha+\beta=1$, Eq. (38) reduces to the following one:

$$
I^{\alpha}(\rho, \Phi)+J^{\alpha}(\rho, \Phi)=\frac{1}{2} \operatorname{Tr}\left[\Phi^{\dagger}(\rho)+\Phi(\rho)\right], \quad 0 \leq \alpha \leq 1
$$

In particular, when $\Phi$ is a unital quantum operation, i.e., $\sum_{i} K_{i}^{\dagger} K_{i}=I$ and $\sum_{i} K_{i} K_{i}^{\dagger}=I$, we get

$$
I^{\alpha}(\rho, \Phi)+J^{\alpha}(\rho, \Phi)=1, \quad 0 \leq \alpha \leq 1 .
$$

which could be viewed as a class of conservation relations. 
In a similar manner, we can investigate the relationship between $V^{\alpha, \beta}(\rho, \Phi)$ and $W^{\alpha, \beta}(\rho, \Phi)$. Define the following quantity

$$
D^{\alpha, \beta}(\rho, K)=\operatorname{Tr}\left(\frac{\rho^{1-\alpha}+\rho^{1-\beta}}{2} K^{\dagger} \frac{\rho^{\alpha}+\rho^{\beta}}{2} K\right), \quad \alpha, \beta \geq 0, \alpha+\beta \leq 1 .
$$

It follows from Eqs. (19) and (20) that

$$
\begin{aligned}
V^{\alpha, \beta}(\rho, K)= & \frac{1}{4}\left[\operatorname{Tr}\left(\rho^{1-\alpha-\beta} K^{\dagger}\left(\frac{\rho^{\alpha}+\rho^{\beta}}{2}\right)^{2} K+K\left(\frac{\rho^{1-\alpha}+\rho^{1-\beta}}{2}\right)\left(\frac{\rho^{\alpha}+\rho^{\beta}}{2}\right) K^{\dagger}\right)\right. \\
& \left.-2 D^{\alpha, \beta}(\rho, K)\right]
\end{aligned}
$$

and

$$
\begin{aligned}
W^{\alpha, \beta}(\rho, K)= & \frac{1}{4}\left[\operatorname{Tr}\left(\rho^{1-\alpha-\beta} K^{\dagger}\left(\frac{\rho^{\alpha}+\rho^{\beta}}{2}\right)^{2} K+K\left(\frac{\rho^{1-\alpha}+\rho^{1-\beta}}{2}\right)\left(\frac{\rho^{\alpha}+\rho^{\beta}}{2}\right) K^{\dagger}\right)\right. \\
& \left.+2 D^{\alpha, \beta}(\rho, K)\right] .
\end{aligned}
$$

Noting that

$$
D^{\alpha, \beta}(\rho, K)=\frac{1}{4}\left(\left\|\rho^{\frac{\alpha}{2}} K \rho^{\frac{1-\alpha}{2}}\right\|_{2}^{2}+\left\|\rho^{\frac{\beta}{2}} K \rho^{\frac{1-\beta}{2}}\right\|_{2}^{2}+\rho^{\frac{\alpha}{2}} K \rho^{\frac{1-\beta}{2}}\left\|_{2}^{2}+\rho^{\frac{\beta}{2}} K \rho^{\frac{1-\alpha}{2}}\right\|_{2}^{2}\right) \geq 0,
$$

we have

$$
V^{\alpha, \beta}(\rho, K) \leq W^{\alpha, \beta}(\rho, K)
$$

and thus

$$
V^{\alpha, \beta}(\rho, \Phi) \leq W^{\alpha, \beta}(\rho, \Phi) .
$$

For any channel $\Phi$ with Kraus representation in the form of Eq. (10), the quantities $V^{\alpha, \beta}(\rho, \Phi)$ and $W^{\alpha, \beta}(\rho, \Phi)$ can be rewritten as

$$
\begin{aligned}
V^{\alpha, \beta}(\rho, \Phi)= & \frac{1}{4} \operatorname{Tr}\left[\rho^{1-\alpha-\beta} \Phi^{\dagger}\left(\left(\frac{\rho^{\alpha}+\rho^{\beta}}{2}\right)^{2}\right)+\Phi\left(\left(\frac{\rho^{1-\alpha}+\rho^{1-\beta}}{2}\right)\left(\frac{\rho^{\alpha}+\rho^{\beta}}{2}\right)\right)\right. \\
& \left.-2\left(\frac{\rho^{1-\alpha}+\rho^{1-\beta}}{2}\right) \Phi^{\dagger}\left(\frac{\rho^{\alpha}+\rho^{\beta}}{2}\right)\right]
\end{aligned}
$$

and

$$
\begin{aligned}
W^{\alpha, \beta}(\rho, \Phi)= & \frac{1}{4} \operatorname{Tr}\left[\rho^{1-\alpha-\beta} \Phi^{\dagger}\left(\left(\frac{\rho^{\alpha}+\rho^{\beta}}{2}\right)^{2}\right)+\Phi\left(\left(\frac{\rho^{1-\alpha}+\rho^{1-\beta}}{2}\right)\left(\frac{\rho^{\alpha}+\rho^{\beta}}{2}\right)\right)\right. \\
& \left.+2\left(\frac{\rho^{1-\alpha}+\rho^{1-\beta}}{2}\right) \Phi^{\dagger}\left(\frac{\rho^{\alpha}+\rho^{\beta}}{2}\right)\right],
\end{aligned}
$$

which implies that for $\alpha, \beta \geq 0, \alpha+\beta \leq 1$,

$$
\begin{aligned}
& V^{\alpha, \beta}(\rho, \Phi)+W^{\alpha, \beta}(\rho, \Phi) \\
& =\frac{1}{4} \operatorname{Tr}\left[\rho^{1-\alpha-\beta} \Phi^{\dagger}\left(\left(\frac{\rho^{\alpha}+\rho^{\beta}}{2}\right)^{2}\right)+\Phi\left(\left(\frac{\rho^{1-\alpha}+\rho^{1-\beta}}{2}\right)\left(\frac{\rho^{\alpha}+\rho^{\beta}}{2}\right)\right)\right] .
\end{aligned}
$$


Furthermore, when $\alpha=\beta=\frac{1}{2}$, Eq. (41) reduces to

$$
V^{\frac{1}{2}, \frac{1}{2}}(\rho, \Phi)+W^{\frac{1}{2}, \frac{1}{2}}(\rho, \Phi)=\frac{1}{2} \operatorname{Tr}\left[\Phi^{\dagger}(\rho)+\Phi(\rho)\right] .
$$

In particular, when $\Phi$ is a unital quantum operation, i.e., $\sum_{i} K_{i}^{\dagger} K_{i}=I$ and $\sum_{i} K_{i} K_{i}^{\dagger}=I$, we obtain

$$
V^{\frac{1}{2}, \frac{1}{2}}(\rho, \Phi)+W^{\frac{1}{2}, \frac{1}{2}}(\rho, \Phi)=1,
$$

which could also be regarded as a conservation relation. Note that from the definitions, $V^{\frac{1}{2}, \frac{1}{2}}(\rho, K)=I(\rho, K)$ and $W^{\frac{1}{2}, \frac{1}{2}}(\rho, K)=J(\rho, K)$, and thus $V^{\frac{1}{2}, \frac{1}{2}}(\rho, \Phi)=I(\rho, \Phi)$ and $W^{\frac{1}{2}}, \frac{1}{2}(\rho, \Phi)=J(\rho, \Phi)$, Eqs. (42) and (43) hold naturally since they are in accord with Eqs. (32) and (33) in Ref. [43, respectively.

Inspired by Ref. [43, we now give two interpretations of the conservation relations Eq. (40) derived above. First of all, for any group representation $\{U(g): g \in G\}$ on a finite group $G$, we can define a quantum channel

$$
\Phi(\rho)=\frac{1}{|G|} \sum_{g \in G} U(g) \rho U(g)^{\dagger}
$$

where $|G|$ denotes the number of elements in $G$. For a compact Lie group $G$, it can be also defined that

$$
\Phi(\rho)=\int \mathrm{d} \mu(g) U(g) \rho U(g)^{\dagger},
$$

where $U(g)$ is the unitary representation of $g \in G$ and $\mathrm{d} \mu$ is the Haar measure with respect to $G$. In this regard, it is natural to interpret $J^{\alpha}(\rho, \Phi)$ and $I^{\alpha}(\rho, \Phi)$ as symmetry and asymmetry of $\rho$ with respect to the group $G$, respectively. Therefore, Eq. (40) could be viewed as a family of symmetry-asymmetry complementarity relations.

Secondly, we illustrate Eq. (40) with wave-particle duality in the Mach-Zehnder interferometry with a path detector [70]. The beam-splitter, mirror and phase shift are realized by the following unitary matrices

$$
U_{B}=\frac{1}{\sqrt{2}}\left(\begin{array}{cc}
1 & 1 \\
1 & -1
\end{array}\right), \quad U_{M}=\left(\begin{array}{ll}
0 & 1 \\
1 & 0
\end{array}\right), \quad U_{\theta}=\left(\begin{array}{cc}
e^{i \theta} & 0 \\
0 & 1
\end{array}\right),
$$

respectively, while the path detector is realized by the unitary matrix $V$. The input state is a bipartite state $\rho^{a b}=\rho \otimes \tau$, where $\rho=\frac{1}{2}(\mathbf{1}+\mathbf{r} \cdot \sigma)$ is the initial (external) system state and $\tau$ is the initial (internal) state. Here $\mathbf{r}=\left\{r_{1}, r_{2}, r_{3}\right\}$ is the Bloch vector with $|\mathbf{r}|^{2} \leq 1$ and $\sigma=\left\{\sigma_{1}, \sigma_{2}, \sigma_{3}\right\}$ with $\sigma_{i}(i=1,2,3)$ the Pauli matrices. The interferometry channel is

$$
\Phi(\rho)=\operatorname{Tr}_{b}\left(U(\rho \otimes \tau) U^{\dagger}\right)
$$

where $U=U_{B}^{a b} U_{M}^{a b} V^{a b} U_{B}^{a b}, U_{B}^{a b}=U_{B} \otimes \mathbf{1}^{b}, U_{M}^{a b}=U_{M} \otimes \mathbf{1}^{b}$ and $V^{a b}=e^{i \theta}|0\rangle\langle 0| \otimes \mathbf{1}^{b}+$ 
$|1\rangle\langle 1| \otimes V$. Direct calculation shows that

$$
\begin{aligned}
I^{\alpha}(\rho, \Phi)= & \frac{1}{4}\left[2-\left(\lambda_{1}^{1-\alpha}+\lambda_{2}^{1-\alpha}\right)\left(\lambda_{1}^{\alpha}+\lambda_{2}^{\alpha}\right)+\frac{\left(\lambda_{1}^{1-\alpha}-\lambda_{2}^{1-\alpha}\right)\left(\lambda_{1}^{\alpha}-\lambda_{2}^{\alpha}\right)}{|r|^{2}} r_{1}^{2}\right. \\
& \left.-\frac{\left(\lambda_{1}^{1-\alpha}-\lambda_{2}^{1-\alpha}\right)\left(\lambda_{1}^{\alpha}-\lambda_{2}^{\alpha}\right)}{|r|^{2}}\left(1-r_{1}^{2}\right)|\operatorname{Tr}(V \tau)| \cos (\theta-\nu-\gamma)\right],
\end{aligned}
$$

and

$$
\begin{aligned}
J^{\alpha}(\rho, \Phi)= & \frac{1}{4}\left[2+\left(\lambda_{1}^{1-\alpha}+\lambda_{2}^{1-\alpha}\right)\left(\lambda_{1}^{\alpha}+\lambda_{2}^{\alpha}\right)-\frac{\left(\lambda_{1}^{1-\alpha}-\lambda_{2}^{1-\alpha}\right)\left(\lambda_{1}^{\alpha}-\lambda_{2}^{\alpha}\right)}{|r|^{2}} r_{1}^{2}\right. \\
& \left.+\frac{\left(\lambda_{1}^{1-\alpha}-\lambda_{2}^{1-\alpha}\right)\left(\lambda_{1}^{\alpha}-\lambda_{2}^{\alpha}\right)}{|r|^{2}}\left(1-r_{1}^{2}\right)|\operatorname{Tr}(V \tau)| \cos (\theta-\nu-\gamma)\right]
\end{aligned}
$$

where $\nu=\arg (\operatorname{Tr}(V \tau)), \gamma=\arctan \frac{2 r_{2} r_{3}}{r_{2}^{2}-r_{3}^{2}}$ and $\lambda_{1,2}=(1 \mp|\mathbf{r}|) / 2$ are the eigenvalues of the qubit state $\rho=\frac{1}{2}(\mathbf{1}+\mathbf{r} \cdot \sigma)$.

Since $I^{\alpha}(\rho, \Phi)$ is a manifestation of the asymmetry, by minimizing over the phase shift, the quantity

$$
\begin{aligned}
\tilde{P}^{\alpha}(\rho, \Phi)= & \min _{\theta} I^{\alpha}(\rho, \Phi) \\
= & \frac{1}{4}\left[2-\left(\lambda_{1}^{1-\alpha}+\lambda_{2}^{1-\alpha}\right)\left(\lambda_{1}^{\alpha}+\lambda_{2}^{\alpha}\right)+\frac{\left(\lambda_{1}^{1-\alpha}-\lambda_{2}^{1-\alpha}\right)\left(\lambda_{1}^{\alpha}-\lambda_{2}^{\alpha}\right)}{|r|^{2}} r_{1}^{2}\right. \\
& \left.-\frac{\left(\lambda_{1}^{1-\alpha}-\lambda_{2}^{1-\alpha}\right)\left(\lambda_{1}^{\alpha}-\lambda_{2}^{\alpha}\right)}{|r|^{2}}\left(1-r_{1}^{2}\right)|\operatorname{Tr}(V \tau)|\right]
\end{aligned}
$$

gives a quantification for the which-path information. In contrast, since $J^{\alpha}(\rho, \Phi)$ represents the symmetry, by maximizing over the phase shift, the quantity

$$
\begin{aligned}
\tilde{W}^{\alpha}(\rho, \Phi)= & \max _{\theta} J^{\alpha}(\rho, \Phi) \\
= & \frac{1}{4}\left[2+\left(\lambda_{1}^{1-\alpha}+\lambda_{2}^{1-\alpha}\right)\left(\lambda_{1}^{\alpha}+\lambda_{2}^{\alpha}\right)-\frac{\left(\lambda_{1}^{1-\alpha}-\lambda_{2}^{1-\alpha}\right)\left(\lambda_{1}^{\alpha}-\lambda_{2}^{\alpha}\right)}{|r|^{2}} r_{1}^{2}\right. \\
& \left.+\frac{\left(\lambda_{1}^{1-\alpha}-\lambda_{2}^{1-\alpha}\right)\left(\lambda_{1}^{\alpha}-\lambda_{2}^{\alpha}\right)}{|r|^{2}}\left(1-r_{1}^{2}\right)|\operatorname{Tr}(V \tau)|\right]
\end{aligned}
$$

could be exploited as a measure of the interference visibility. Utilizing these quantities with parameter $\alpha$, we thus obtain a family of complementarity relations signifying the wave-particle duality:

$$
\tilde{P}^{\alpha}(\rho, \Phi)+\tilde{W}^{\alpha}(\rho, \Phi)=1, \quad 0 \leq \alpha \leq 1
$$

which is more general than Eq. (43) in Ref. 43].

\section{Conclusions and discussions}


We have showed that the state-channel interactions based on modified generalized Wigner-Yanase-Dyson skew information could be exploited as a family of coherence measures with respect to a quantum channel under certain assumptions. We have also provided explicit analytical expressions of this family of coherence measures for qubit states with respect to various kinds of quantum channels. Moreover, complementarity relations based on MGWYD skew information and MWGWYD skew information have been derived. Inspired by Ref. [43], we have also presented two interpretations of the conservation relations: the interplay between symmetry and asymmetry with respect to a group, and the wave-particle duality.

Coherence and complementarity are both fundamental issues in quantum mechanics and quantum information theory. As is shown in Ref. [43], the quantities $I(\rho, \Phi)$ and $J(\rho, \Phi)$ not only present a basic and an alternative framework for addressing complementarity, but also put forward the study of coherence in a broad context involving quantum channels. The quantity $I(\rho, \Phi)$ has several interpretations including asymmetry, coherence, incompatibility, quantumness and quantum uncertainty with state-channel interactions, while $J(\rho, \Phi)$ possesses the corresponding interpretations in a dual fashion. We have revisited the work of Refs. 43] and [44] based on the interplay of $I^{\alpha, \beta}(\rho, \Phi)$ and $J^{\alpha, \beta}(\rho, \Phi)$ as well as $V^{\alpha, \beta}(\rho, \Phi)$ and $W^{\alpha, \beta}(\rho, \Phi)$ induced by MGWYD skew information and MWGWYD skew information, and investigated the coherence and complementarity based on measures with parameters $\alpha$ and $\beta$. Therefore, our results are valid for a large family of quantities, which are more general than the ones induced by MWY skew information and MWYD skew information proposed in Ref. [43] and Ref. [4], respectively.

The quantities in Eqs. (8) and (9) are defined in terms of the Hilbert-Schmidt norm. A natural question arises: could $I(\rho, \Phi)=\sum_{i}\left\|\left[\rho^{\frac{1}{2}}, K_{i}\right]\right\|_{p}^{2}$ be exploited as a measure of coherence with respect to a quantum channel? Or more generally, could $I(\rho, \Phi)=$ $\sum_{i}\|\|\left[\rho^{\frac{1}{2}}, K_{i}\right] \|\left.\right|^{2}$ be utilized as a measure of coherence with respect to a quantum channel for other unitarily invariant norms other than Schatten $p$-norms? These questions deserve further investigations.

\section{Acknowledgements}

This work was supported by National Natural Science Foundation of China (Grant Nos. 11701259, 11971140, 11461045, 11675113, 11301124), the China Scholarship Council (Grant No.201806825038), the Key Project of Beijing Municipal Commission of Education under No. KZ201810028042, Beijing Natural Science Foundation (Z190005), Natural Science Foundation of Zhejiang Province of China (LY17A010027). This work was completed while Zhaoqi Wu and Lin Zhang were visiting Max-Planck-Institute for Mathematics in the Sciences in Germany. 


\section{References}

[1] Baumgratz, T., Cramer, M. and Plenio, M. B.: Quantifying coherence, Phys. Rev. Lett. 113, 140401 (2014)

[2] Yuan, X., Zhou, H., Cao, Z. and Ma, X.: Intrinsic randomness as a measure of quantum coherence, Phys. Rev. A 92, 022124 (2015)

[3] Winter, A. and Yang, D.: Operational resource theory of coherence, Phys. Rev. Lett. 116, 120404 (2016)

[4] Napoli, C., Bromley, T. R., Cianciaruso, M., Piani, M., Johnston, N. and Adesso, G.: Robustness of Coherence: An operational and observable measure of quantum coherence, Phys. Rev. Lett. 116, 150502 (2016)

[5] Singh, U., Zhang, L., Pati, A. K.: Average coherence and its typicality for random pure states. Phys. Rev. A 93, 032125 (2016)

[6] Zhang, L.: Average coherence and its typicality for random mixed quantum states, J. Phys. A Math. Theor. 50, 155303 (2017)

[7] Chen, B., Fei, S.-M.: Notes on modified trace distance measure of coherence. Quantum Inf. Process. 17, 107 (2018)

[8] Bu, K., Singh, U., Fei, S.-M., Pati, A. K., Wu, J.: Maximum relative entropy of coherence: an operational coherence measure, Phys. Rev. Lett. 119, 150405 (2017)

[9] Yu, C.-S.: Quantum coherence via skew information and its polygamy, Phys. Rev. A 95, 042337 (2017)

[10] Sun, Y., Mao, Y. and Luo, S.: From quantum coherence to quantum correlations, Europhys. Lett. 118, 60007 (2017)

[11] Luo, S. and Sun, Y.: Quantum coherence versus quantum uncertainty, Phys. Rev. A 96, 022130 (2017)

[12] Luo, S. and Sun, Y.: Partial coherence with application to the monotonicity problem of coherence involving skew information, Phys. Rev. A 96, 022136 (2017)

[13] Jin, Z.-X., Fei, S.-M.: Quantifying quantum coherence and nonclassical correlation based on Hellinger distance, Phys. Rev. A 97, 062342 (2018)

[14] Xiong, C., Kumar, A., Wu, J.: Family of coherence measure and duality between quantum coherence and path distinguishability, Phys. Rev. A 98, 032324 (2018)

[15] Zhu, X.-N.,Jin, Z.-X., Fei, S.-M.: Quantifying quantum coherence based on the generalized $\alpha$-z-relative Rényi entropy, Quantum Inf. Process. 18, 179 (2019) 
[16] Zanardi, P., Styliaris, G., Venuti, L. C.: Coherence-generating power of quantum unitary maps and beyond, Phys. Rev. A 95, 052306 (2017)

[17] Zanardi, P., Styliaris, G., Venuti, L. C.: Measures of coherence-generating power for quantum unital operations, Phys. Rev. A 95, 052307 (2017)

[18] Zhang, L., Ma, Z.,Chen, Z., Fei, S.-M.: Coherence generating power of unitary transformations via probabilistic average, Quantum Inf. Process. 17, 186 (2018)

[19] Streltsov, A., Singh, U., Dhar, H. S., Bera, M. N. and Adesso, G.: Measuring quantum coherence with entanglement, Phys. Rev. Lett. 115, 020403 (2015)

[20] Radhakrishnan, C., Parthasarathy, M., Jambulingam, S., Byrnes, T.: Distribution of quantum coherence in multipartite systems, Phys. Rev. Lett. 116, 150504 (2016)

[21] Ma, J., Yadin, B., Girolami, D., Vedral, V., Gu, M.: Converting coherence to quantum correlations, Phys. Rev. Lett. 116, 160407 (2016)

[22] Chitambar, E., Hsieh, M. H.: Relating the resource theories of entanglement and quantum coherence, Phys. Rev. Lett. 117, 020402 (2016)

[23] Zhu, H., Ma, Z., Cao, Z., Fei, S.-M., Vedral, V.: Operational one-to-one mapping between coherence and entanglement measures, Phys. Rev. A 96, 032316 (2017)

[24] Piani, M., Cianciaruso, M., Bromley, T. R., Napoli, C., Johnston, N., Adesso, G.: Robustness of asymmetry and coherence of quantum states, Phys. Rev. A 93, 042107 (2016)

[25] Marvian, I., Spekkens, R. W., Zanardi, P.: Quantum speed limits, coherence, and asymmetry, Phys. Rev. A 93, 052331 (2016)

[26] Bu, K., Anand N. and Singh, U.: Asymmetry and coherence weight of quantum states, Phys. Rev. A 97, 032342 (2018)

[27] Lostaglio M. and Müller, M. P.: Coherence and asymmetry cannot be broadcast, Phys. Rev. Lett. 123, 020403 (2019)

[28] Marvian I. and Spekkens, R. W.: No-broadcasting theorem for quantum asymmetry and coherence and a trade-off relation for approximate broadcasting, Phys. Rev. Lett. 123, 020404 (2019)

[29] Tan, K. C., Choi, S., Jeong, H.: Optimizing nontrivial quantum observables using coherence, New. J. Phys. 21, 023013 (2019)

[30] Bischof, F., Kampermann, H. and Bruß, D.: Resource theory of coherence based on positive-operator-valued measures, Phys. Rev. Lett. 123, 110402 (2019) 
[31] Theurer, T., Egloff, D., Zhang, L., Plenio, M. B.: Quantifying operations with an application to coherence, Phys. Rev. Lett. 122, 190405 (2019)

[32] Bohr, N.: Das Quantenpostulat und die neuere Entwicklung der Atomistik, Naturwissenschaften 16, 245 (1928); The Quantum postulate and the recent development of atomic theory, Nature (London) 121, 580 (1928)

[33] Heisenberg, W.: The Physical Principles of the Quantum Theory, The University of Chicago Press, Chicago (1930)

[34] Englert, B.-G.: Fringe visibility and which-way information: an inequality, Phys. Rev. Lett. 77, 2154 (1996)

[35] Dürr S., Nonn, T. and Rempe, G.: Fringe visibility and which-way information in an atom interferometer, Phys. Rev. Lett. 81, 5705 (1998)

[36] Peng, X., Zhu, X., Suter, D., Du, J., Liu, M. and Gao, K.: Quantification of complementarity in multiqubit systems, Phys. Rev. A 72, 052109 (2005)

[37] Tang, J.-S., Li, Y.-L., Li, C.-F. and Guo, G.-C.: Revisiting Bohr's principle of complementarity with a quantum device, Phys. Rev. A 88, 014103 (2013)

[38] Selleri, F.: Wave-Particle Duality, Springer, Berlin (2012)

[39] Coles, P. J., Kaniewski, J. and Wehner, S.: Equivalence of wave-particle duality to entropic uncertainty, Nat. Commun. 5, 5814 (2014)

[40] Coles, P. J.: Entropic framework for wave-particle duality in multipath interferometers, Phys. Rev. A 93, 062111 (2016)

[41] Coles, P. J., Berta, M., Tomamichel, M. and Wehner, S.: Entropic uncertainty relations and their applications, Rev. Mod. Phys. 89, 015002 (2017)

[42] Bagan, E., Calsamiglia, J., Bergou J. A. and Hillery, M.: A generalized wave-particle duality relation for finite groups, J. Phys. A: Math. Theor. 51, 414015 (2018)

[43] Luo, S., Sun, Y.: Coherence and complementarity in state-channel interaction, Phys. Rev. A 98, 012113 (2018)

[44] Li, W., Monotonnicity of skew information and its applications in quantum resource theory, Quantum Inf. Process. 18, 166 (2019)

[45] Chen, P., Luo S.: Direct approach to quantum extensions of Fisher information, Front. Math. China 2, 359 (2007)

[46] Nielsen, M. A., Chuang, I. L.: Quantum Computation and Quantum Information, Cambridge University Press, Cambridge (2000) 
[47] Wigner, E. P., Yanase, M. M.: Information contents of distributions, Proc. Natl. Acad. Sci. USA 49, 910-918 (1963)

[48] Furuichi, S., Yanagi, K., Kuriyama, K.: Trace inequalities on a generalized WignerYanase skew information, J. Math. Anal. Appl. 356, 179 (2009)

[49] Luo, S.: Wigner-Yanase skew information and uncertainty relations, Phys. Rev. Lett. 91, 180403 (2003)

[50] Luo, S.: Quantum uncertainty of mixed states based on skew information, Phys. Rev. A 73, 022324 (2006)

[51] Luo, S., Zhang, Q.: Skew information decreases under quantum measurements, Theor. Math. Phys. 151, 529 (2007)

[52] Luo, S.: Notes on superadditivity of Wigner-Yanase-Dyson information, J. Stat. Phys. 128, 1177 (2007)

[53] Cai, L., Luo, S.: On convexity of generalized Wigner-Yanase-Dyson information, Lett. Math. Phys. 83, 253 (2008)

[54] Cai, L., Li, N., Luo, S.: Weak superadditivity of skew information, J. Phys. A 41, 135301 (2008)

[55] Furuichi, S.: Schrödinger uncertainty relation with Wigner-Yanase skew information, Phys. Rev. A 82, 034101 (2010)

[56] Furuichi, S.: Inequalities for Tsallis relative entropy and generalized skew information, Linear Multilinear Algebra 59, 1143 (2011)

[57] Chen, B., Fei, S.-M., Long, G.: Sum uncertainty relations based on Wigner-Yanase skew information, Quantum Inf. Process. 15, 2639 (2016)

[58] Ma, Z., Chen, Z., Fei, S.-M.: Uncertainty relations based on skew information with quantum memory, Sci. China Phys. Mech. Astron. 60, 010321 (2017)

[59] Long, G.L.: General quantum interference principle and duality computer. Commun. Theor. Phys. 45, 825 (2006)

[60] Bender, C. M. and Boettcher, S.: Real spectra in non-Hermitian Hamiltonians having PT symmetry. Phys. Rev. Lett. 80, 5243 (1998)

[61] Makris, K. G., El-Ganainy, R., Christodoulides, D. and Musslimani, Z. H.: Beam dynamics in PT symmetric optical lattices. Phys. Rev. Lett. 100, 103904 (2008)

[62] Guo, A., Salamo, G., Duchesne, D., Morandotti, R., Volatier-Ravat, M., Aimez, V., Siviloglou, G. and Christodoulides, D.: Observation of PT-symmetry breaking in complex optical potentials. Phys. Rev. Lett. 103, 093902 (2009) 
[63] Ruter, C. E., Makris, K. G., El-Ganainy, R., Christodoulides, D. N., Segev, M. and Kip, D. Observation of parity-time symmetry in optics. Nat. Phys. 6, 192 (2010)

[64] Chang, L., Jiang, X., Hua, S.,Yang, C., Wen, J., Jiang, L., Li, G., Wang, G. and Xiao, M.: Parity-time symmetry and variable optical isolation in active-passivecoupled microresonators. Nat. Photon. 8, 524 (2014)

[65] Tang, J.-S., Wang, Y.-T., Yu, S., He, D.-Y., Xu, J.-S., Liu, B.-H., Chen, G., Sun, Y.-N., Sun, K., Han, Y.-J., Li, C.-F. and Guo G.-C.: Experimental investigation of the no-signalling principle in parity-time symmetric theory using an open quantum system. Nature Photon. 10, 642 (2016)

[66] Dou, Y., Du, H.: Generalizations of the Heisenberg and Schrödinger uncertainty relations, J. Math. Phys. 54, 103508 (2013)

[67] Dou, Y., Du, H.: Note on the Wigner-Yanase-Dyson skew information, Int. J. Theor. Phys. 53, 952 (2014)

[68] Chen, Z., Liang, L., Li, H., Wang, W.: Two generalized Wigner-Yanase skew information and their uncertainty relations, Quantum Inf. Process. 15, 5107 (2016)

[69] Bhatia, R.: Matrix analysis, Springer, Berlin (2013)

[70] Sjöqvist, E., Pati, A. K., Ekert, A., Anandan, J. S., Ericsson, M., Oi, D. K. L. and Vedral, V.: Geometric phases for mixed states in interferometry, Phys. Rev. Lett. $85,2845(2000)$ 